\title{
Research Report In Vitro Assay for Site-Specific Proteases Using Bead-Attached GFP Substrate
}

\author{
Daksha Patel, John Frelinger, \\ Jaap Goudsmit ${ }^{1,2}$, and \\ Baek Kim \\ University of Rochester, \\ Rochester, NY, USA, \\ ${ }^{1}$ University of Amsterdam, \\ and ${ }^{2}$ Amsterdam Institute of \\ Viral Genomics, Amsterdam, \\ The Netherlands
}

\section{ABSTRACT}

Site-specific proteases, which catalyze cleavage of peptide bonds in specific amino acid sequences of target proteins, play important roles in various biological events of many living organisms. In humans, disruption in regulation of these site-specific proteases can lead to pathological consequences. Here, we report a simple in vitro assay for enzymatic activities of site-specific proteases. This assay system employs a protein substrate molecule that is comprised of (i) His-tag binding module, (ii) cleavage sites, and (iii) green fluorescent protein (GFP) detection module. In this study, prostate-specific antigen (PSA) and Thrombin-specific cleavage sites were introduced into the substrate molecules. The overexpressed GFP substrate protein was purified with the aid of $\mathrm{Ni}^{++}$-charged magnetic beads. On cleavage by either PSA or Thrombin, GFP was released from the bound magnetic beads, enabling a direct measurement of the cleaved product by fluorescence. Detection sensitivity, as well as the kinetics of reaction of PSA cleavage with the GFP substrate, was similar or better than commercially available PSA fluorogenic peptide substrate. This bead-attached GFP substrate was also used for an inhibition assay using a competitive inhibitor of Thrombin. In conclusion, this as- say offers a simple fluorescent method for monitoring the activity of the site-specific proteases. Furthermore, this system provides flexible means of incorporating varying sizes of flanking sequences adjacent to the cleavage site, which can be essential for studying the regulatory macromolecular interactions between proteases and their substrates.

\section{INTRODUCTION}

Site-specific proteases, which cleave peptide bonds contained in specific amino acid sequences of the target polypeptides, have been identified in many organisms, including viruses, bacteria, and humans. These site-specific proteases are key molecules that perform post-translational processing of the target polypeptides involved in various types of biological events. Some site-specific proteases found in multicellular organisms are secreted from the cells and migrate to process extra-cellular target polypeptide molecules.

Recently, in humans, several sitespecific proteases that are associated with specific diseases have been identified. For example, prostate specific antigen (PSA), a serine protease that normally cleaves kinen peptides involved in sperm mobility, is elevated at abnormally high levels in the blood of the prostate cancer patients $(17,19)$, and PSA expression level is used as a biomarker for the diagnosis of prostate cancer. Also, recent identification of an Alzheimer-specific aspartic protease suggests a possible role for this sitespecific protease in physiological and pathological events of Alzheimer's disease $(21,22)$. Human metalolloproteinases (MMP), which cleave mem- brane bound proteins such as integrins, are thought to be involved in various biological events such as tumor invasion, metastasis, and angiogenesis $(4,15,23)$. Site-specific proteases involved in blood clotting, such as Factor Xa and Thrombin, have been extensively studied $(2,20)$. Finally, human immunodeficiency virus (HIV-1) site-specific protease has been studied for its biological role in the pathogenesis of HIV and for its biochemical properties to evaluate structure-function relationships $(6,10,12)$.

The availability of in vitro enzymatic assays for these site-specific proteases enables biochemical characterization of the proteases and their substrates as well as efficient screening for protease inhibitors. Fluorogenic peptide substrates to assay proteolytic activity of proteases with diversified cleavage sequences are widely used $(8,11,14)$. However, these substrates are typically short peptides ( $2-5$ amino acids long) and, therefore, limit studies involving characterization of the large native form of the substrate. As demonstrated in recent studies of site-specific proteases involved in blood coagulation $(9,16)$, the use of large-size polypeptide substrates in cleavage reactions with respective site-specific proteases have revealed extended macromolecular interactions between enzyme exosites and regulatory regions of native protein substrates, which control biological and physiological functions of proteolysis. For example, protease activated receptor 1 (PAR1), which is a membrane-bound receptor substrate of Thrombin, contains a regulatory sequence (hirudinlike) near the Thrombin cleavage site, which can interact with the protease exosites and control proteolysis (9). Thus, 
a protease assay that allows the inclusion of a longer polypeptide substrate would be advantageous for screening protease inhibitors that would compete against natural substrates under biologically relevant conditions. However, unlike a short peptide substrate with convenient probes such as a fluorogenic moiety, protease assays for macro-protein substrates heavily rely on either chromatographic separation techniques or SDS-PAGE analysis for the size alteration of the substrates. Recently, an assay using enhanced green fluorescent protein (EGFP) fluorescence to detect cleavage by Thrombin has been reported (18). However, this assay has a major limitation-insolubility of the substrate, which limits its utility.

Here, we report an improved in vitro proteolytic assay system to study enzymatic activities of site-specific proteases involving a polypeptide substrate with resin/bead-attached GFPuv protein. Also, we tested utility of our system by fusing protease cleavage sequences of clinically significant proteases, PSA and Thrombin, to the GFPuv molecule. This GFPuv assay system not only solved the solubility problem but also significantly improved the sensitivity of the assay for the detection of the cleavage products. Cleavage kinetics and the detection sen-

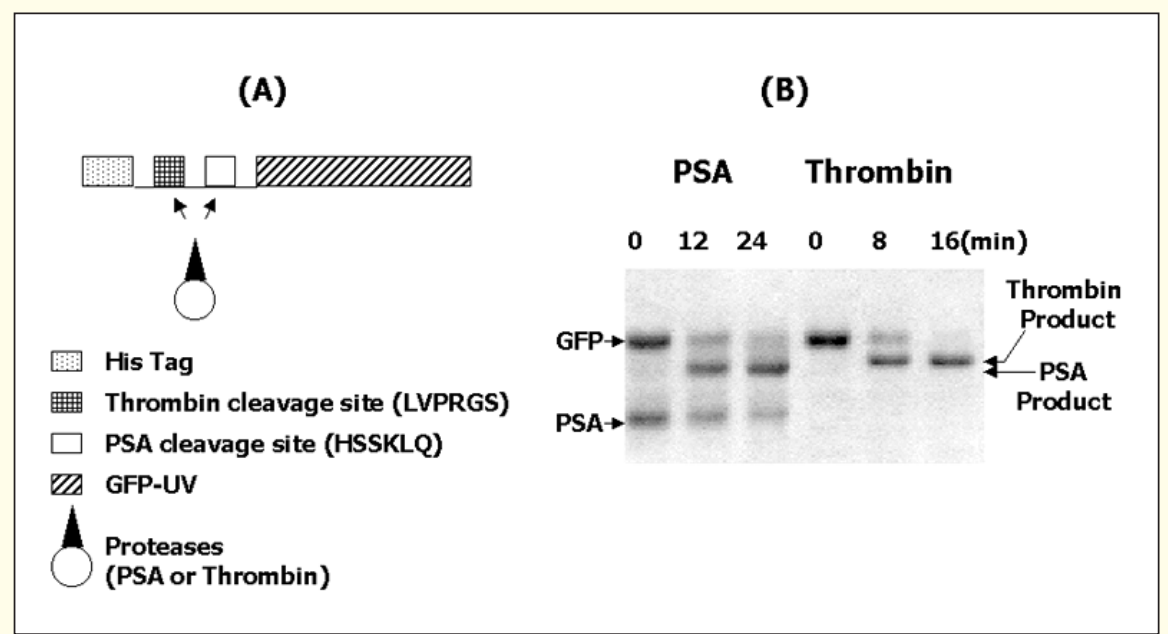

Figure 1. GFP substrate molecule and its cleavage reaction by PSA. (A) Constructed GFP substrate molecule. This GFP substrate is composed of (i) binding module (His-tag), (ii) cleavage module (Thrombin and PSA cleavage sites), and (iii) detection module (GFPuv). Proteolytic reaction at the cleavage module of the constructed GFP substrate separates the binding module and detection module. The amino acid positions between the binding and detection modules were marked. (B) Cleavage of the GFP substrate by PSA and Thrombin. One microgram of purified GFP substrate was cleaved by PSA ( $1 \mu \mathrm{g})$ and Thrombin $(0.04 \mathrm{U})$ in $20 \mu \mathrm{L}$ reaction at $37^{\circ} \mathrm{C}$, and the reaction was analyzed by $12 \%$ SDS-PAGE. Cleavage of the intact 28-kDa GFP substrate by PSA and Thrombin generates 25- and 26-kDa cleavage products, respectively, that have lost the binding module.

sitivity of this GFP substrate were equal or higher compared to the fluorogenic peptide substrate. More importantly, with this GFP-based substrate, it is pose to incorporate longer polypeptides which coulde their flanking sequences, factors in proteolysis.

\section{METHODS AND MATERIALS}

\section{Construction of Plasmid Expressing GFP Substrate Molecule}

As shown in Figure 1A, the GFP substrate protein is comprised of three functional modules: $(i)$ a binding module with a 6 His tag, (ii) a cleavage module with both the PSA cleavage site (this study) and the Thrombin cleavage site (pET28c; Novagen, Madison, WI, USA), and (iii) a detection module with a derivative of green fluorescent protein called GFPuv (BD Biosciences (lto, CA, USA). GFPmore fluorescent under UV light than wild-type GFP (3). To construct the plasmid expressing the GFP substrate, pBK121, the 0.7-kb GFPuv gene and the 26-bp PSA cleavage site (5) were inserted into Bam HI/XhoI sites and the

1196 BioTechniques
NheI site of pET28c, respectively. The GFP gene was amplified from pGFPuv (BD Biosciences Clontech) using 5' GFPuv forward primer ( $5^{\prime}$-TTTTTGGATCCATGAGTAAAGGAGAAGAACTTTTCAC-3': the start codon is in bold, and the BamHI site is underlined) and 3' GFPuv reverse primer (5'-TTTTTCTCGAGTTATTTGTAGAGCTCATCC-3': the termination codon is in bold, and the XhoI site is underlined). Oligomers encoding top (5'-TTTTTTGCTAGCCATTCTTCTAAATTGCAAGCTAGCTTTTTT-3') and bottom (5'-AAAAAAGCTAGCTTGCAATTTAGAAGAATGGCTAGCAAAAAA$\left.3^{\prime}\right)$ strands of PSA cleavage sequences (HSSKLQ, bold) with NheI (underlined) flanking sequences were annealed before digestion (NheI) and ligation. A GFP construct containing two PSA cleavages at the NheI site was also obtained during this cloning procedure. The amino acid sequence of the PSA and Thrombin cleavage sites in the constructed GFP substrate is shown in Figure $1 \mathrm{~A}$. This construct has a 40-aminoacid-long cleavage module between the binding and detection modules.

\section{Purification of GFP Substrate}

pBK121 expresses the substrate protein with a 6 His tag, Thrombin cleavage site, and GFPuv in E. coli. It was transformed into bacterial strain BL21(DE3), and the protein was overexpressed by $0.1 \mathrm{mM}$ IPTG induction in $2 \times$ YT media $(500 \mathrm{~mL})$ with kanamycin $(30 \mu \mathrm{g} / \mathrm{mL})$ at $0.1 \mathrm{~A}_{600}$, for $2 \mathrm{~h}$. The cells were harvested at $5600 \times$ $g$ and resuspended into $10 \mathrm{~mL} 1 \times$ binding buffer (Novagen). The cell pellet was snap-frozen and thawed on ice for $1 \mathrm{~h}$ for lysis. The lysed cells were pelleted by centrifugation at $14000 \times g$ for $20 \mathrm{~min}$, and the supernatant was collected. Overexpressed protein was purified from the supernatant either by $\mathrm{Ni}^{++}$-charged resin (Novagen) or $\mathrm{Ni}^{++}$ charged magnetic beads (Qiagen, Valencia, CA, USA). Purification was carried out at $4^{\circ} \mathrm{C}$ using Novagen reagents. For column purification, $5 \mathrm{~mL}$ cell lysate supernatant was applied to the pre-charged $\mathrm{Ni}^{++}$column $(1.5 \mathrm{~mL})$. The resin was washed three times with $1 \times$ binding solution and twice with a 70:30 mixture of $1 \times$ binding and $1 \times$ 
washing solutions (5 mL/washing). Protein bound to the resin was eluted with $5 \mathrm{~mL} 1 \times$ elution buffer, and five 1 $\mathrm{mL}$ aliquots of fraction were collected. Fractions were analyzed for the presence of the purified protein by green fluorescence and SDS-PAGE analysis. The fractions showing bright fluorescence under UV light and expected size of the substrate $(28 \mathrm{kDa})$ were pooled and dialyzed overnight with $1 \times$ dialysis buffer without DTT $(0.1 \mathrm{mM})$, followed by dialysis for $8 \mathrm{~h}$ with $1 \times$ dialysis buffer with DTT. The protein concentration was determined using the Bradford assay (Bio-Rad Laboratories, Hercules, CA, USA). The purified protein yield was $0.5 \mathrm{mg} / 500 \mathrm{~mL}$ cell culture. The GFP substrate containing two PSA cleavage sites was also purified.

\section{Magnetic Bead Purification}

Cells from a $10-\mathrm{mL}$ induced culture were resuspended in $1 \mathrm{~mL}$ lysis buffer, lysed, and spun. The green fluorescent supernatant was rotated for $10 \mathrm{~min}$ with $100 \mu \mathrm{L} 5 \%$ suspension of $\mathrm{Ni}^{++}$ charged magnetic beads at $4{ }^{\circ} \mathrm{C}$. The bound protein was rapidly precipitated with the magnetic bar and washed twice with $1 \times$ binding buffer and twice with a 70:30 mixture of $1 \times$ binding and $1 \times$ washing solutions ( $1 \mathrm{~mL} /$ washing). Under UV light, the beads appeared green and the supernatant appeared clear after separation (Figure 2).

\section{Cleavage Reaction of Bead-Attached GFP Substrate}

Purified GFP substrate protein was cleaved by two site-specific proteases, PSA (Calbiochem-Novabiochem, San Diego, CA, USA) and Thrombin (0.4 $\mathrm{U} / \mu \mathrm{L}$; Novagen). PSA or Thrombin was incubated with purified GFP substrate or bead-attached GFP substrate at $37^{\circ} \mathrm{C}$ in respective $1 \times$ buffers (Tris- $\mathrm{HCl}, \mathrm{pH}$ 7.5, $200 \mathrm{mM} \mathrm{NaCl}$ for PSA; Thrombin $1 \times$ buffer from Novagen). For comparison of the reaction rates, PSA fluorogenic peptide substrate (Mu-HSSKLQAFC; Calbiochem-Novabiochem) containing identical cleavage site as the GFP substrate was also used (Figure 3). Concentrations of substrate and enzymes are shown in the Figure 3 legend. The concentration of the peptide sub- strate $(4 \mu \mathrm{M})$ used in this assay is much lower than its $\mathrm{K}_{\mathrm{m}}$ value $(470 \mu \mathrm{M})(5)$. For the experiment shown in Figure 3, two aliquots $(20 \mu \mathrm{L})$ were taken at each time point from a total of $400 \mu \mathrm{L}$ reaction containing the reagents described above. The reaction mixture with the bead-attached GFP substrate was mixed intermittently during the reaction. The reaction mixture was applied to a magnetic bar for $5 \mathrm{~s}$ to precipitate the beads containing the uncleaved GFP substrate, and aliquots of supernatant with the cleaved GFP were taken at set time points. Aliquots taken during the reaction were snap-frozen in a dry-ice ethanol bath. In the reaction with the GFP substrate, no further cleavage reaction can occur in the aliquots taken because the aliquots taken contain only cleaved substrate. Therefore, unlike the reactions with the peptide substrate, the termination of protease reaction in the GFP sample aliquots before analysis was not necessary, which is an additional advantage of the method.

\section{Inhibition by Competitive Inhibitor of Thrombin}

Inhibition of the Thrombin cleavage reaction with bead-attached GFP sub- strate was performed using a Thrombin inhibitor (Tosyl-G-P-R-4-nitranilide acetate; Roche Molecular Biochemicals, Indianapolis, IN, USA). The cleavage reaction $(200 \mu \mathrm{L})$ of the GFP substrate $(1 \mu \mathrm{M})$ by Thrombin $(0.4 \mathrm{U})$ was performed as described above in the presence $(200 \mu \mathrm{M})$ or absence of the Thrombin inhibitor.

\section{Detection of Reaction Products}

PSA- or Thrombin-cleaved GFP supernatant aliquots $(20 \mu \mathrm{L})$ were spotted on a UV light box (Figure 3A) for visual determination or diluted in $30 \mu \mathrm{L}$ cold water (final $50 \mu \mathrm{L}$ ) and quantified for fluorescence using a FluoroCount fluorometer (Packard Instrument, Meriden, CT, USA; $505 \mathrm{~nm}$ for the emission wavelength; Figure 3, B and C). For controls, known amounts of GFP $(1 \mu \mathrm{M}$ in $50 \mu \mathrm{L})$ and fluorogenic peptide substrate (4 $\mu \mathrm{M}$ in $50 \mu \mathrm{L}$; CalbiochemNovabiochem) were fully cleaved with an excess amount of PSA $(0.5 \mu \mathrm{g} / 10$ $\mu \mathrm{L}$ ) during a 2-h incubation. We found that the peptide substrate requires four times higher concentration than the GFP substrate to obtain a similar level of fluorescence intensity by the fluorometer.

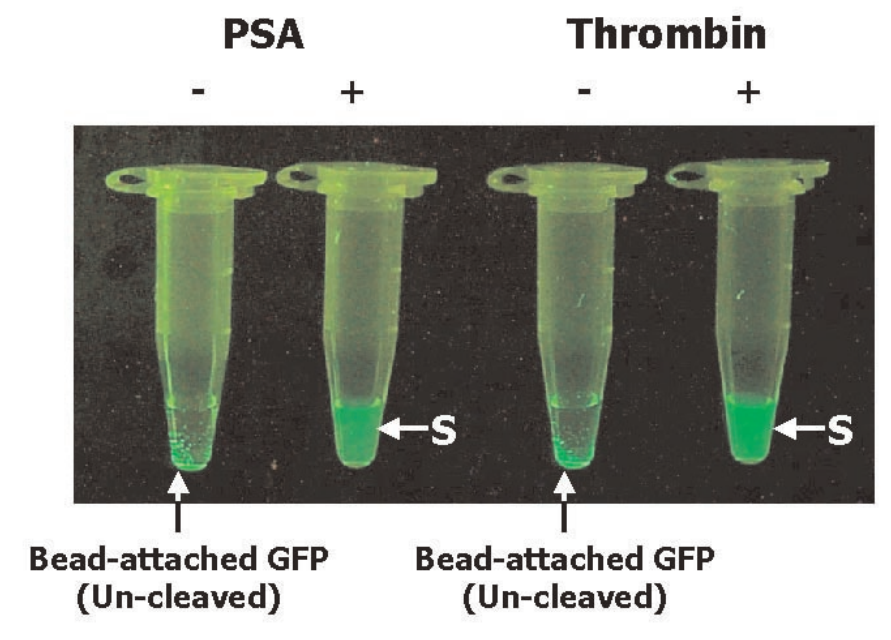

Figure 2. Proteolytic cleavage of bead-attached GFP substrate by PSA and Thrombin. Bead-attached GFP substrate $(0.5 \mu \mathrm{g} / 20 \mu \mathrm{L})$ was incubated in the presence (+) or absence (-) of PSA $(0.5 \mu \mathrm{g})$ or Thrombin $(0.02 \mathrm{U})$ at $37^{\circ} \mathrm{C}$ for $1 \mathrm{~h}$. Cold water $(100 \mu \mathrm{L})$ was added to the reaction, and the beads were precipitated by a magnetic bar and exposed to UV. Cleavage by Thrombin or PSA results in release of GFP into the supernatant (S). In reactions without Thrombin or PSA, uncleaved GFP remains in the bead fraction. 


\section{RESULTS}

\section{Cleavage of Purified GFP Substrate by PSA and Thrombin}

To determine proteolytic cleavage of the constructed GFP substrate (Figure 1A) by PSA and Thrombin, cleavage reactions of purified GFP with PSA and Thrombin were carried out and analyzed using SDS-PAGE. As seen in Figure $1 \mathrm{~B}$, cleavage of the GFP substrate by PSA and Thrombin generated shorter substrate products and disappearance of the full-length substrate. Western analysis with $\mathrm{Ni}^{++}$-conjugated alkaline phosphatase (Ni-NTA-AP; Qiagen) showed that the full-length sub- strate interacts with Ni-NTA-AP, whereas the cleaved product does not (data not shown), confirming that cleavage of the GFP substrate by PSA removes the His-tag attached to the Nterminal of the GFP substrate.

\section{Cleavage Detection with Bead- Attached GFP Substrate}

Next, the magnetic bead-attached GFP substrate was analyzed for proteolytic processing of the PSA cleavage site. Overexpressed GFP substrate protein from bacterial lysate was captured by $\mathrm{Ni}^{++}$-charged magnetic beads (see Materials and Methods) and precipitated from the lysate by a magnetic bar. The precipitated beads were fluorescent, whereas the supernatant was clear (see uncleaved GFP in -PSA of Figure 2). On incubation with either thrombin or PSA, magnetic bead-attached GFP substrate was cleaved and released into the supernatant. After $1 \mathrm{~h}$ of incubation, the cleavage reaction was complete, as seen by the appearance of green fluorescence in the supernatant (Figure 2) and the loss of green fluorescence on the precipitated magnetic beads. This observation suggests that, unlike the EGFP substrate previously reported (18), the GFP cleavage products are completely soluble, which allows accurate measurements for the portions of cleaved (in supernatant) and uncleaved (attached to the

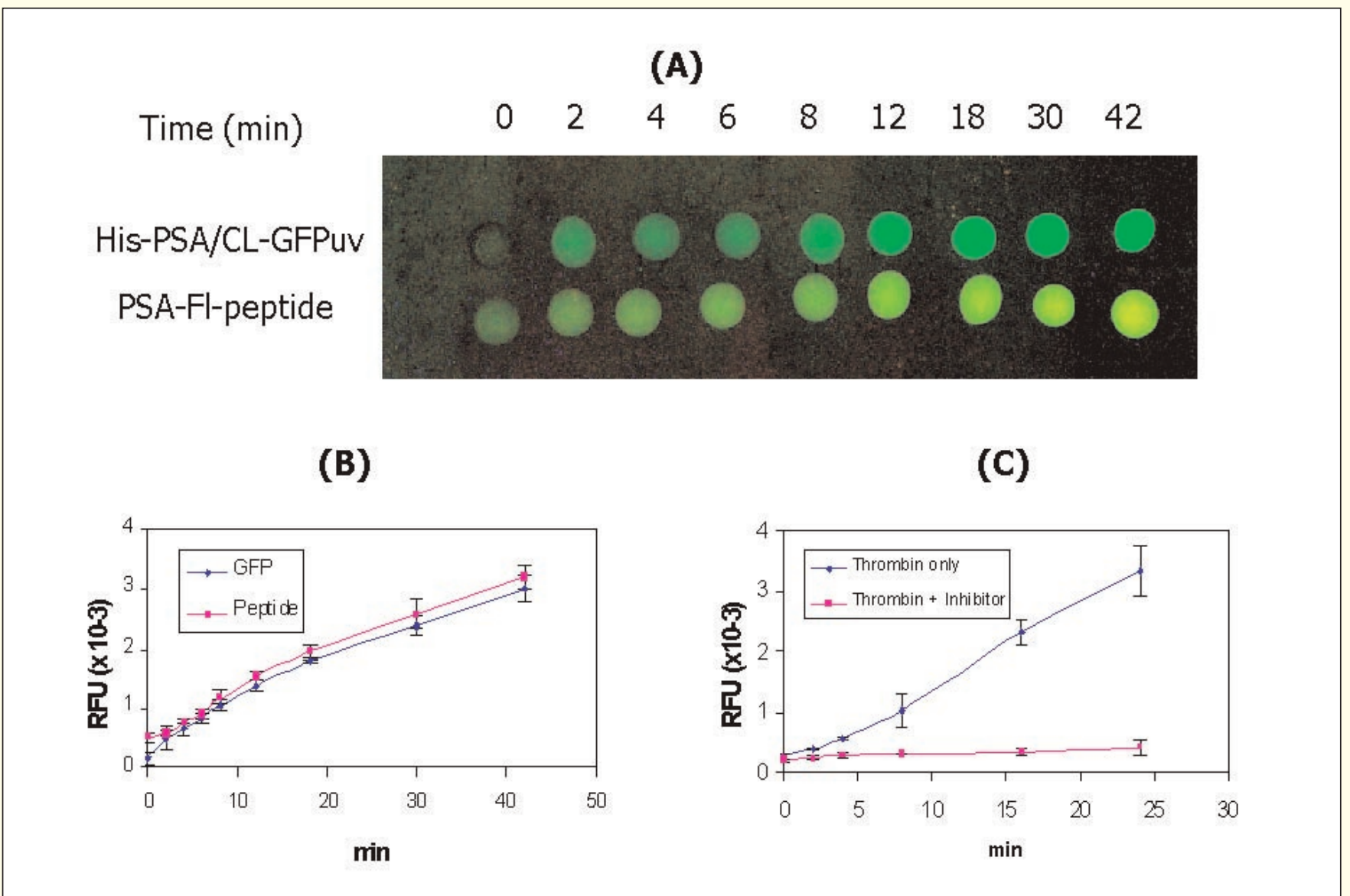

Figure 3. Comparison of PSA cleavage reaction with GFP substrate and fluorogenic peptide substrate and detection of inhibition of Thrombin cleavage reaction by a competitive peptide inhibitor. Bead-attached GFP protein $(1 \mu \mathrm{M})$ or fluorogenic peptide $(4 \mu \mathrm{M})$ was incubated with PSA (10 $\mu \mathrm{g})$ in $400 \mu \mathrm{L}$ reaction. At each time point, two $20-\mu \mathrm{L}$ aliquots (in case of GFP substrate, beads were rapidly separated from the supernatant before being frozen) were snapfrozen and stored until analysis. Completely cleaved GFP substrate $(1 \mu \mathrm{M}$ in $50 \mu \mathrm{L})$ and fluorogenic peptide substrates $(4 \mu \mathrm{M}$ in $50 \mu \mathrm{L})$ gave 5.3 and $6.1 \times 10^{3}$ RFU (Packard Instrument), respectively, under this experimental condition. (A) Visualization by UV light. Aliquots (20 $\mu \mathrm{L})$ were spotted on a UV light box, and the fluorescing GFP and peptide in the aliquots were visualized by UV light. (B) Fluorometric analysis. Aliquots were applied to a fluorometer, and the fluorescence intensity in each aliquot taken at different time points was plotted. (C) Inhibition of Thrombin cleavage reaction by a peptide inhibitor. The Thrombin $(0.2 \mathrm{U}$ in $200 \mu \mathrm{L})$ cleavage reaction of the bead-attached GFP substrate $(1 \mu \mathrm{M})$ was performed in the presence and absence of a Thrombin peptide inhibitor $(200$ $\mu \mathrm{M})$ as described above. Aliquots were taken at different time points, and the amount of cleaved GFP protein was measured by a fluorometer. Data in Figure 3, $\mathrm{B}$ and $\mathrm{C}$, were compiled from multiple replicates of the reactions. 
beads) GFP substrate. The presence of the cleaved GFP product in the supernatant and their absence in the magnetic beads were confirmed by SDS-PAGE (data not shown).

\section{Comparison of Reaction Rates with Bead-Attached GFP Substrate and Fluorogenic Peptide Substrate}

Fluorogenic peptide substrates have been used for enzymatic analysis of some site-specific proteases. Proteolytic processing of this peptide generates a cleavage product that emits green fluorescence. Next, we compared the reaction rates of PSA with the bead-attached GFP substrate $(1 \mu \mathrm{M})$ and a PSA fluorogenic peptide $(4 \mu \mathrm{M})$. The substrate concentrations used in this experiment are much lower than the $\mathrm{K}_{\mathrm{m}}$ value of the peptide substrate $(470 \mu \mathrm{M})(5)$. Aliquots were taken at various time points after initiation of the cleavage reaction by PSA, and cleavage products were analyzed by both visual detection with UV light (Figure 3A) and a fluorometer (505 $\mathrm{nm}$; Figure 3B). GFP and peptide substrates digested to completion were used as controls for the determination of reaction rates. We found that four times higher concentration of the peptide substrate compared to the GFP substrate was needed for equal fluorescence intensity as measured by the fluorometer. As seen in Figure 3, A and B, the cleavage reaction rate with the two substrates was similar under conditions in which the fluorescent cleavage product could be detected by the instrument. The linearity of the reaction rate was slightly decreased in the longer time-points (Figure 3B), which might result from a high level of fluorescence (high concentration of the cleavage product) that was beyond the linear ranges of the fluorescence detection of the fluorometer. Dilution of the reaction solutions containing high concentrations of GFP cleavage products is recommended for extending the linearity of this protease assay.

\section{Inhibition of Thrombin Cleavage Reaction by a Peptide Inhibitor}

Time-course cleavage reaction of the bead-attached GFP substrate with Thrombin in the presence or absence of a Thrombin peptide inhibitor was per- formed. As seen in Figure 3C, the relative fluorescence value (RFU) of the supernatant that was supposed to contain cleaved GFP substrate remained undetectable for the +Thrombin inhibitor reaction, indicating inhibition of proteolytic cleavage by the inhibitor.

\section{DISCUSSION}

Here, we report an improved protease assay for the analysis of site-specific proteases involving three different modules: (i) binding module (His-tag), (ii) protease cleavage module, and (iii) detection module (GFPuv). The cleavage kinetics and the detection sensitivity of this GFP substrate are higher compared to the PSA fluorogenic peptide substrate. This assay offers several advantages over existing protocols in the analysis of proteases. These include the solubility of the substrate and the use of GFPuv as the cleavage detection module. Since the rate of cleavage and release of GFPuv can be measured directly by a fluorometer, this assay obviates the need for time-consuming SDSPAGE/western analysis. Also, in cases where the cleavage site is at the extreme end of the C-terminal of a protein, it would be difficult to detect size differences after cleavage on SDS-PAGE or western blot analyses. Furthermore, the GFPuv substrate offers a wide use of applications because the molecule does not have size restrictions. For example, it would be possible to, in principle, determine the physical interactions between full-length native (or large-size) polypeptide substrates and proteases. Recent studies involving Thrombin and its receptor substrate, called PAR1, have revealed important interactions between enzyme exosites and sequences distal to the cleavage sites of the substrate, as cofactors in the regulation of proteolysis (9). Therefore, an assay that does not limit substrate size would be advantageous in reconstituting biologically relevant proteolytic reactions. In this study, the GFP substrate tested contains a 40amino-acid-long cleavage site between binding and detection modules, which is considerably longer than conventional fluorescent peptide substrates (approximately 10 amino acids or less) such as the 6-amino-acid-long PSA peptide con- tained in the current study. Therefore, the thrombin cleavage site and the hirudin-like regulatory sequence of PAR1 substrate, which is less than 40 amino acids (9), could conceivably be incorporated in the module to study regulatory macromolecular interactions between thrombin and PAR1. It is known that GFPuv can be fused to varying sizes of polypeptides without loss of biochemical properties such as green fluorescence and solubility (7). In addition, GFP can be fused to the N-terminal end of the molecules $(1,13)$, instead of the $\mathrm{C}$ terminal in the cases where physical interference occurs between exosites and the C-terminal GFP tag. Because of the flexibility of DNA recombination techniques, the cleavage sites and their flanking sequences can be easily modified. For example, different sizes of the flanking sequences can be incorporated, and mutations in both cleavage and regulatory flanking sequences can be introduced. Finally, it is possible to insert multiple copies of the same cleavage sites or combination cleavage sequences to enhance the kinetic sensitivity and versatility of this protease assay system.

\section{ACKNOWLEDGMENTS}

This work was supported by Amsterdam Support Diagnostics b.v. to B.K. and National Institutes of Health grant no. CA70218 to J.F.

\section{REFERENCES}

1.Chen, J. and P. Ahlquist. 2000. Brome mosaic virus polymerase-like protein $2 \mathrm{a}$ is directed to the endoplasmic reticulum byhelicase-like viral protein 1a. J. Virol. 74:4310-4318.

2.Coughlin, S.R. 2000. Thrombin signaling and protease-activated receptors. Nature 407:258264.

3.Crameri, A., E.A. Whitehorn, E. Tate, and W.P. Stemmer. 1996. Improved green fluorescent protein by molecular evolution using DNA shuffling. Nat. Biotechnol. 14:315-319.

4.Curran, S. and G.I. Murray. 1999. Matrix metalloproteinases in tumor invasion and metastasis. J. Pathol. 189:300-308.

5.Denmeade, S.R., W. Lou, J. Lovgren, J. Malm, H. Lilja, and J.T. Isaacs. 1997. Specific and efficient peptide substrates for assaying the proteolytic activity of prostate-specific antigen. Cancer Res. 57:4924-4930.

6.Eron, J.J., Jr. 2000. HIV-1 protease inhibitors. Clin. Infect. Dis. 30(Suppl 2):S160-S170.

7.Gerdes, H.H. and C. Kaether. 1996. Green 
fluorescent protein: applications in cell biology. FEBS Lett. 389:44-47.

8.Harris, J.L., B.J. Backes, F. Leonetti, S. Mahrus, J.A. Ellman, and C.S. Craik. 2000. Rapid and general profiling of protease specificity by using combinatorial fluorogenic substrate libraries. Proc. Natl. Acad. Sci. USA 97: 7754-7759.

9.Jacques, S.L., M. LeMasurier, P.J. Sheridan, S.K. Seeley, and A. Kuliopulos. 2000. Substrate-assisted catalysis of the PAR1 thrombin receptor. J. Biol. Chem. 275:40671-40678.

10.Krafft, G.A. and G.T. Wang. 1994. Synthetic approaches to continuous assays of retroviral proteases. Methods Enzymol. 241:70-86.

11.Lamb, M.L., K.W. Burdick, S. Toba, M.M. Young, A.G. Skillman, X. Zou, J.R. Arnold, and I.D. Kuntz. 2001. Design, docking, and evaluation of multiple libraries against multiple targets. Proteins 42:296-318.

12.Lebon, F. and M. Ledecq. 2000. Approaches to the design of effective HIV-1 protease inhibitors. Curr. Med. Chem. 7:455-477.

13.Lecerf, J.M., T.L. Shirley, Q. Zhu, A. Kazantsev, P. Amersdorfer, D.E. Housman, A. Messer, and J.S. Huston. 2001. Human single-chain Fv intrabodies counteract in situ huntingtin aggregation in cellular models of Huntington's disease. Proc. Natl. Acad. Sci. USA 98:4764-4769.

14.Mattler, L.E. and N.U. Bang. 1977. Serine protease specificity for peptide chromogenic substrates. Thromb. Haemost. 38:776-792.

15.McQuibban, G.A., J.H. Gong, E.M. Tam, C.A. McCulloch, I. Clark-Lewis, C.M. Overall. 2000. Inflammation dampened by gelatinase A cleavage of monocyte chemoattractant protein-3. Science 289:1202-1206.

16.Myles, T., B.F. Le Bonniec, and S.R. Stone. 2001. The dual role of thrombin's anion-binding exosite-I in the recognition and cleavage of the protease-activated receptor 1. Eur. J. Biochem. 268:70-77.

17.Okabe, E., J. Kajihara, Y. Usami, and K. Hirano. 1999. The cleavage site specificity of human prostate specific antigen for insulin-like growth factor binding protein-3. FEBS Lett. 447:87-90.

18.Pehrson, J.C., A. Weatherman, J. Markwell, G. Sarath, and S.D. Schwartzbach. 1999. Use of GFP as a reporter for the facile analysis of sequence-specific proteases. BioTechniques 27:28-32.

19.Rittenhouse, H.G., J.A. Finlay, S.D. Mikolajczyk, and A.W. Partin. 1998. Human Kallikrein 2 (hK2) and prostate-specific antigen (PSA): two closely related, but distinct, kallikreins in the prostate. Crit. Rev. Clin. Lab. Sci. 35:275-368.

20.Scarborough, R.M. 1998. Coagulation factor $\mathrm{Xa}$ : the prothrombinase complex as an emerging therapeutic target for small molecule inhibitors. J. Enzyme Inhib. 14:15-25.

21.Sinha, S. and I. Lieberburg. 1999. Cellular mechanisms of $\beta$-amyloid production and secretion. Proc. Natl. Acad. Sci. USA 96:1104911053.

22.Steinhilb, M.S., R.S. Turner, and J.R. Gaut. 2001. The protease inhibitor, MG132, blocks maturation of the amyloid precursor protein Swedish mutant preventing cleavage by $\beta$-secretase. J. Biol. Chem. 276:4476-4484
23.Stracke, J.O., A.J. Fosang, K. Last, F.A. Mercuri, A.M. Pendas, E. Llano, R. Perris, P.E. Di Cesare et al. 2000. Matrix metalloproteinases 19 and 20 cleave aggrecan and carti lage oligomeric matrix protein (COMP). FEBS Lett. 478:52-56.

Received 28 February 2001; accepted 13 June 2001.

\section{Address correspondence to:}

Dr. Baek Kim

601 Elmwood Avenue, Box 672

Department of Microbiology and Immunology

University of Rochester Medical Center

Rochester, NY 14672, USA

e-mail: baek_kim@urmc.rochester.edu 